\title{
Corner Detection for Intelligent Vehicles Based on a Four-Layer Radar
}

\author{
Hongquan Liu, Mingyue Feng, Mingxi Li, Zhichao Zhang \\ Military Transportation University, TianJin, China \\ e-mail: fmy1980@yahoo.cn
}

Keywords: intelligent vehicle, corner detection, road edge.

\begin{abstract}
Intelligent vehicle is thought to be an ideal way to deal with transportation problems. Corner detection is an important yet tough problem for intelligent vehicle because more complicated pictures may be generated by visions or radars from corners than from straight roads. Four-Layer radar was selected for this task for its ability in detecting roads in more abominable natural conditions than machine vision. Radar mapping feature was firstly analyzed, and road corner trait was described based on the radar map. Road edge points as well as smoothness of road surface were the two selected characteristics to find out the road scope, and road width varying extent was the primary benchmark for corners judging. Radar data from all the four layers were combined to overcome problems possibly occur with a fast vehicle or a narrow corner. Experimental results verified a high accuracy rate of the promoted method in corner recognizing.
\end{abstract}

\section{Introduction}

Modern transportation system has brought with plenty of benefits to our city life, yet many disadvantages also occurred simultaneously such as traffic jam, traffic accidents et al. Intelligent vehicle, also called unmanned vehicle, is regarded as a promising means to deal with these traffic problems in the future. Although many progresses have been obtained on intelligent vehicle research in recent years, many crucial technical issues are preventing it from being practically applied in real traffic framework. Road corner detection is such an issue which aims at finding out the front corner from the road in time for the vehicle to determine its next action. Vehicle crash or turning position missing may occur if the problem of road corner detection is not perfectly resolved.

Many efforts have been done on the corner detection problem recently. Edward Rosten summed up former researches on corner detection, and classified detection methods into two types, say edges-based detection, greylevel derivative for corner recognizing as well as detection using direct greylevel information ${ }^{[1]-[2]}$. A high-speed corner detection method based on machine learning was promoted, the repeatability and efficiency of which was verified. In the research, corner was regarded as a graphical mutation on an image. Bandera analyzed a common approach based on curvature function calculation of the contour from an image, and designed a method preventing too much noisy from the curvature function of the approach ${ }^{[3]-[4]}$. Above literatures concentrated on finding out figurate corners on images which is not restricted on road corners. By comparison, fewer researches exactly on road corner detection could be found. Larsson researched on intersection finding for an underground mining vehicle ${ }^{[5]}$. A laser radar was adopted to detect consecutive road surface and walls, and intersection was recognized once the consecutiveness was broken. Things become different when outside environment is considered. University of Karlsruhe developed an intelligent vehicle which took part in 2007 DARPA Urban Challenge, and the corner detection task was completed by lane-marks finding using an onboard camera ${ }^{[6]-[7]}$. Dae-Woon Lim concentrated on judging passing permission at an intersection, basis not only include corner detection, but also contain violation signals ${ }^{[8]}$. Jiuxiang $\mathrm{Hu}^{[9]}$ and $\mathrm{Kin}$ Leong $\mathrm{Ho}^{[10]}$ researched on the corner detection problem from a macroscopical view using large-scaled maps from aerial photograph.

In this paper, a four-layer laser radar is adopted to detect roads ahead of a vehicle, and a road corner detection algorithm is designed based on road width varying extent. 


\section{Radar Detecting Pattern and Corner Imaging Trait}

The four-layer radar sends out radiation in four planes simultaneously by a rotating mirror inside and generates four scanning lines after receiving reflections from every detected positions from the four planes. In our vehicle, the radar is mounted on the middle-top in the front, and Fig.1 shows the detecting way of the radar.

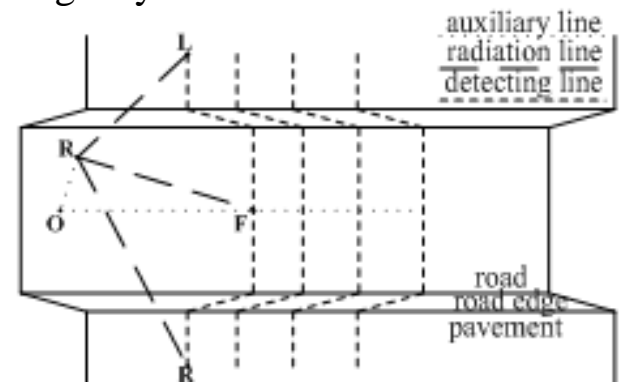

Fig. 1. Radar detecting pattern

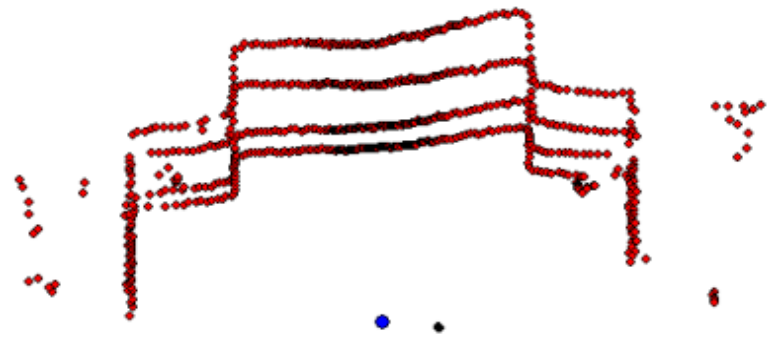

Fig.2 Radar data in Cartesian coordinate

In Fig.1, $\mathrm{R}$ is the place of the radar and $\mathrm{O}$ is its projection on the ground. $\mathrm{L}, \mathrm{F}$ and $\mathrm{R}$ represent the detected points on the left boundary, the right middle point and the right boundary respectively, and then other detected points between $\mathrm{L}$ and $\mathrm{R}$ are depicted by a detecting line. The four detecting lines are typically parallel with a distance that could be calculated by mathematical functions between any two neighbored ones. Eq. 1 is used to calculate the distance $d_{i, i+1}$ between the $i^{\text {th }}$ and the $i+1^{\text {th }}$ lines.

$$
d_{i, i+1}=\overline{O R} \times \tan \left(\angle F_{i+1} R O+\Delta \theta\right)-\overline{O R} \times \tan \left(\angle F_{i} R O\right)
$$

$\overline{O R}$ is the height of the radar, $\angle F_{1} R O$ is with a constant angle value once the radar is fixed, and $\Delta \theta$ is a fixed angle that is valued about $1.07^{\circ}$ by our Alasca XT radar.

By converting the detecting picture into a Cartesian coordinate, we get Fig.2 in which the middle-down point is the vehicle and the four lines are formed by discrete detected points. Fig. 2 shows the vehicle running on a straight road with no corners in the front, and the four detecting lines may become more irregular once the vehicle is coming into a corner. Fig.3 shows the process of the vehicle passing through a corner.

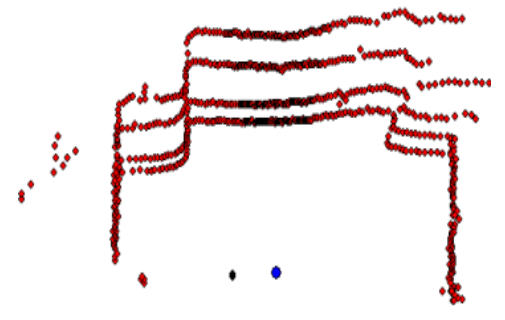

3-1 entering a corner

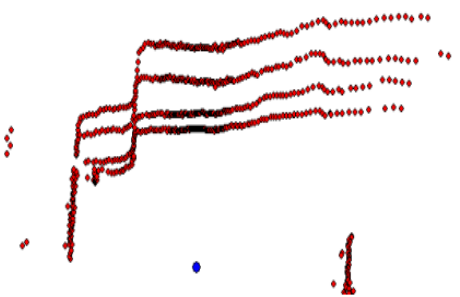

3-2 passing through a corner

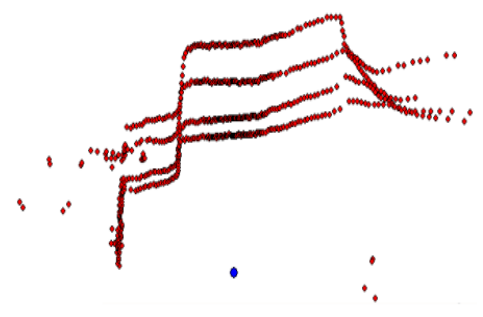

3-3 leaving a corner

Fig. 3. The process of passing through a one-side corner

The following conclusions are drawn for corner traits summarizing based on Fig.3:

(1) A relatively long vertical line may appear in the picture, both sides of which are two long horizontal lines. This vertical line is formed by the road edge that is helpful for us to find out the road square. One issue should be noticed that not all long vertical lines are from road edges, excluding methods should be carried out to assure a high accuracy rate in road finding.

(2) The road line may become longer fast inside the corner than in the narrow road. As is shown in Fig.3-2, the right side of the road is extending with a high percentage, indicating that the right side is inside a corner. Small discontinuousness may occur on the road line because of discrimination between the two intersected roads which needs to be copped with in corner identifying.

(3) When passing through a corner, not all road lines vary their shapes simultaneously. Instead, they are changing one by one with the same mode. As is shown in Fig.3-1 and Fig.3-3, the front line is extending or shrinking its road space at the earliest, and the other lines follows the step of that line with a small time interval.

The upper corner traits are the basis for corner detection algorithm design in the next section. 


\section{Corner Detection Method}

(1) Single road line detecting

Road line detection is the basis for corner detection, and using one detecting line from the four to detect one road line is the first step. Trait (1) of corner introduced in last section is the primary basis for road line finding, and two hypotheses are made for the purpose of simplification. One hypothesis is that the vehicle keeps relatively parallel with the road, meaning the angle between the vehicle direction and the road is small. This hypothesis is to assure that the middle detecting point of the radar falls into the road scope other than on sideways beyond the road. This hypothesis is regarded rational for the reason that once the middle detecting points of the radar is on the outside from the road, meaning that the vehicle is running inclined with the road with a relatively large sloping angle, dangers may possibly occur with the vehicle running outside the road or its lane causing crash with other vehicles. In this situation, manual operations are needed for adjusting. The other hypothesis is that the road surface is relatively smooth and little holes together with scattered small matters appear on the road. This hypothesis is regarded fulfilled in most city or campus transportation environment.

Obeying the two hypotheses, the process of single road line detecting is illustrated as follows:

Step1. Set the middle detecting point as the current searching position, and the left direction as the searching orientation.

Step2. If the remaining number of left points of the current searching position is less then $\mathrm{K}$, go to Step6; else go to Step3.

Step3. Collect the left K neighbored points of the current point as the candidate road edge set, and calculate the $\mathrm{X}$ and the $\mathrm{Y}$ values of each point in the set. Calculate the mean square deviation value of $\mathrm{Y}$ for the set, denoting as $m s d v_{y}$. For $\mathrm{X}$ values, the difference values for every two neighbored point pair in the set are calculated first, and the mean square deviation value of the difference of $X$ are then calculated, noted as $m s d v_{d x}$. Compare $m s d v_{y}$ and $m s d v_{d x}$ with the threshold values $Y_{\text {thres }}$ and $X_{\text {thres. }}$. If $m s d v_{y}>Y_{\text {thres }}$ and $m s d v_{d x}<X_{\text {thres }}$, go to Step5; else, go to step4.

Step4. Set the left neighbored point as the current searching point, and add the searching iteration number by 1 . Go back to Step 2.

Step5. The left road edge is founded, and the point $i$ is regarded as the left road edge point. Go to Step7.

Step6. The left road edge does not exist, and whole left space is regarded as a road. Go to Step7.

Step7. Search the right road edge from Step1 again by changing the searching process rightward.

After the searching process, the left and right ends of the road in both sides could be found out, and the space between the two ends is regarded as road. If the end in one side is not found, then the whole side is regarded as road.

(2) Corner detecting

By trait(2) of road corners, the road length varying extent is a primary trait for corner detection. In this paper, we design two comparison mechanism to assess the road length varying extent. On one hand, the road length from the first layer of the radar detection is compared with its historical values to judge whether a sudden road length variation occurs. On the other hand, the two road lengths from the first and the last layers are compared with each other to judge whether the former is varying fast. The second comparison is based on the assumption that when the first detection line of the radar arrives at a corner, the last line is still on the former road space, keeping a distance with the entrance of the corner. Following calculations are carried out to verify this assumption.

Assume the detecting frequency of the radar $f$ is $20 \mathrm{~Hz}$, and the vehicle is running with a high speed of $s=60 \mathrm{~km} / \mathrm{h}$. Fixed parameters of the radar after being installed on the vehicle include: the height $h=1 \mathrm{~m}$, downward angle(for the last layer) $\theta=75^{\circ}$, and the angle between the first and the last layer $\Delta \theta=3.2^{\circ}$. Then the vehicle moving distance $d_{v}=s / f$ in one detecting period is $0.8333 \mathrm{~m}$, while distance between the ground projections of the first and the last detecting lines $d_{l}=h \times(\tan (\theta+\Delta \theta)-\tan (\theta))=$ $1.0546 \mathrm{~m}$. The two values could ensure that in at least one time period, the first detecting line has entered into the corner while the last line keeps inside the former straight road. 
The corner detecting method is based on the upper two comparisons. Being different with the road line detection restricted in one time node, the corner detecting process is time-dependent. We use the radar detecting period as the calculating period meaning that the algorithm is carried out simultaneously with the radar detection. First assign the starting time $t$ to be 0 , then the algorithm is illustrated as follows:

Step1. Set parameters. Set $K=0$, which represents the times of the first detecting line since possibly entered the corner. Set threshold values $K_{\text {thres }}, D S_{\text {thres }}$ and $D D_{\text {thres }}$ with experience numbers. Assign $h$ with experience numbers too.

Step2. Add $t$ by 1.

Step3. Judge whether in corner-candidate state(CCS), and if so go to Step4, else go to Step6.

Step4. Do the second comparison: compare the road length from the first detecting line with its $h$ th historical value(that is the value in the time of $t-h$ ). If the difference between the two values is larger than $D S_{\text {thres }}$, add $K$ by 1 . Else, assign $K$ to be 0 and set the CCS state to be false, and go back to Step2.

Step5. Judge whether $K$ is larger than $K_{\text {thres. }}$ If so go to Step7, else go back to Step2.

Step6. Do the first comparison: compare the road lengths from the first and the last detecting lines. If the difference is larger than $D D_{\text {thres }}$, set CCS to be true and go back to Step4. Else go back to Step 2.

Step7. A corner is found. Calculate corner parameters such as corner position using triangle relationships shown in Fig.1.

\section{Numerical Result}

To evaluate effectiveness of the method in this paper, we have done amount of experiments in campus roads. By these experiments, we found that the method could be influenced by many factors such as the speed of the vehicle, and curvature of the corner, the width of the incoming corner, the surface quality of the road et al. In this paper, we mainly focus on the first two factors that has been verified the most primary ones by experiments. Selected experiments here were carried out in campus environment with well-conditioned road surface and with moderate road width at about 4 5 meters.

Corners with different curvatures are selected, which are classified into three types, say with small, moderate and large curvatures. Corners with small curvatures have a right-angle-like curvature, while those with larger curvatures have a smooth connection between the two crossed roads with a long road arc. Curvatures of moderate corners lie between the upper two types. The three corner types are denoted as $C_{S}, C_{M}, C_{L}$. Another varying parameter is the vehicle speed. We executed experiments under the speed at $10 \mathrm{~km} / \mathrm{h}, 30 \mathrm{~km} / \mathrm{h}$ and $60 \mathrm{~km} / \mathrm{h}$ respectively, which are denoted as $\mathrm{S}_{\mathrm{S}}, \mathrm{S}_{\mathrm{M}}$, and $\mathrm{S}_{\mathrm{F}}$ respectively. Two measures are used to evaluate the effectiveness of the corner detection method, say real corner detecting accuracy rate and fate corner detecting rate, noted as $\mathrm{M}_{1}$ and $\mathrm{M}_{2}$ respectively. By doing mounts of experiments and calculating average values, the outcomes are shown in Table 1.

Table 1. performance of the corner detecting method

\begin{tabular}{|c|c|c|c|c|c|c|}
\hline \multirow{2}{*}{} & \multicolumn{2}{|c|}{$\mathbf{S}_{\mathbf{S}}$} & \multicolumn{2}{c|}{$\mathbf{S}_{\mathbf{M}}$} & \multicolumn{2}{c|}{$\mathbf{S}_{\mathrm{F}}$} \\
\cline { 2 - 7 } & $\mathbf{M}_{\mathbf{1}}$ & $\mathbf{M}_{\mathbf{2}}$ & $\mathbf{M}_{\mathbf{1}}$ & $\mathbf{M}_{\mathbf{2}}$ & $\mathbf{M}_{\mathbf{1}}$ & $\mathbf{M}_{\mathbf{2}}$ \\
\hline $\mathbf{C}_{\mathbf{L}}$ & 0.7121 & 0.1850 & 0.7351 & 0.1538 & 0.7217 & 0.1094 \\
\hline $\mathbf{C}_{\mathbf{M}}$ & 0.8390 & 0.1789 & 0.8378 & 0.1531 & 0.8415 & 0.1156 \\
\hline $\mathbf{C}_{\mathbf{S}}$ & 0.8867 & 0.1825 & 0.8902 & 0.1492 & 0.8967 & 0.0977 \\
\hline
\end{tabular}

From Table 1, we can get the following conclusions: (1) The curvature of a corner has an apparent influence on the method, and as the curvature decreases, the real corner detecting accuracy rate improves. This is because that for a corner with a small curvature, the arc connection between the front two road segment is short, thus the road length varying speed is fast and the length varying extent is large, helpful for distinguishing the corner with historical straight road. When the corner is with a large curvature, the situation is just on the opposite. One thing should be noted that although a corner with a small curvature is easy to be detected, it is more difficult for the vehicle to do the turning operation. (2) The curvature of a corner has little influence on the fate corner detecting rate 
once key parameters of the method have been fixed. (3) As the speed of the vehicle increases, the fate corner detecting rate decreases with a relatively high extent. This is because we have set a temporary "in corner-candidate state" in detecting a corner in the method(see Step3 in the method). This state could not be arrived if the fate corner is too narrow or the vehicle speed is too fast, avoiding the first and the last detecting lines of the radar fall on different types of roads(fate corner and straight road) simultaneously. (4) The speed of vehicle also has a positive affection on the real corner detecting accuracy rate in most cases. This is because a higher speed makes the road length varying extent more apparent between two consecutive calculating time nodes, helping the detecting method in corner finding. We should notice that once the speed is too large, this regular may be broken, refer to the assumption introduced in the "corner detecting” content in last section.

\section{Conclusions}

Corner detection is a primary difficulty that is hindering the development of intelligent vehicles. A four-layer radar is adopted for the corner detecting task. Three corner traits are summarized first and the corner-passing process is analyzed. The appearance of the road length varying extent is finally adopted for corner recognition and a corner detection method is designed for this task. Experiments are done to verify this method.

\section{Acknowledgment}

The authors gratefully acknowledge Chinese Natural Science Committee for funding projects on intelligent vehicle(project codes: 91120306 and 51175290) for us.

\section{References}

[1] E. Rosten and T. Drummond: In Proceedings of 9th European Conference on Computer Vision (ECCV’06) (Graz, Austria, May 7-13, 2006), p.430.

[2] E. Rosten, R. Porter and T. Drummond: IEEE Transactions on Pattern Analysis and Machine Intelligence, Vol. 32 (2010) No.1, p.105.

[3] A. Bandera, C. Urdiales and F. Arrebola, et al: Electronics Letters, Vol. 36 (2000) No. 2, p.124.

[4] C. Urdiales, C. Trazegnies and A. Bandera, et al: Electronics Letters, Vol. 32 (2003) No.5, p.426.

[5] J. Larsson, M. Broxvall, A. Saffiotti: In Proceedings of the IEEE/RSJ International Conference on Intelligent Robots and Systems (IROS) (Nice, France, 2008), p.2222.

[6] C. Duchow: In Proceedings of the 2005 IEEE Computer Society Conference on Computer Vision and Pattern Recognition (CVPR’05), (San Diego, California June 20-26 2005).

[7] S. Kammel, J. Ziegler, and B. Pitzer, et al: Journal of Field Robotics, Vol. 25 (2008), p.615.

[8] D.W. Lim, S.H. Choi, J.S. Jun: In Proceedings of 5th IEEE International Conference on Image Analysis and Interpretation (Apr. 2002), p.126.

[9] J.X. Hu, A.Razdan, and J.C. Femiani, et al: IEEE Transactions on Geoscience and Remote Sensing, Vol. 45 (2007) No.12, p.4144.

[10] K.L. Ho, P.Newman: In International Conference on Computational Intelligence, Robotics and Autonomous Systems (Singapore, December 2005). 